\title{
Genetic polymorphisms analysis of CYP2D6 in the Uygur population
}

\author{
Xue He ${ }^{1,2}, \mathrm{Na} \mathrm{He}^{1,2}$, Lisong Ren ${ }^{3}$, Yongri Ouyang ${ }^{3}$, Ning Zhang ${ }^{1,2}$, Yini $\mathrm{Ma}^{3}$, Dongya Yuan ${ }^{1,2}$, Longli Kang ${ }^{1,2}$ \\ and Tianbo $\operatorname{Jin}^{1,2,3,4^{*}}$
}

\begin{abstract}
Background: This study aimed to investigate genetic polymorphisms of CYP2D6 among healthy Uygur individuals. Genetic polymorphisms of CYP2D6 could greatly affect CYP2D6 activity and lead to differences among individuals in drug efficacy or side effects. To investigate genetic polymorphisms of CYP2D6 in the Uygur population, we directly sequenced the whole gene in 96 unrelated, healthy Uygur volunteers from the Xinjiang Uygur Autonomous Region and screened for genetic variants in the promoter, intron, exons, and 3'UTR.

Results: We detected 62 genetic polymorphisms of CYP2D6, 16 of which were novel SNP with three novel non-synonymous mutations detected for the first time. The allelic frequencies of CYP2D $*^{*} 1,{ }^{*} 10$, *39, and ${ }^{*} 48$ were $0.542,0.156,0.068,0.229$, and 0.073 , respectively. The frequency of CYP2D $6 * 1 /{ }^{*} 10$ which decreased CYP2D6 enzyme activity was $31.3 \%$.

Conclusions: Our results provided basic information about CYP2D6 polymorphisms, suggested that the enzymatic activities of CYP2D6 might be different within the Uygur ethnic group, and provide a basis for safer drug administration and better therapeutic treatment of Uygur individuals.
\end{abstract}

Keywords: Frequency, Genetic polymorphisms, Phenotypic

\section{Background}

The CYP superfamily is one of the most important enzyme systems involved in the biotransformation of many endogenous and exogenous substances. Cytochrome P450 (CYP450) enzymes are essential for the metabolism of many medications. This class has more than 50 enzymes, among them CYP2D6 is one of the most significant enzymes [1]. The enzyme accounts for only a small percentage of all hepatic P450s, but its role in drug metabolism is extensively higher than its relative content [2]. CYP2D6 is an important polymorphic phase-I drug-metabolism enzyme and plays an important role in the metabolism of a variety of drugs and environmental compounds [3]. It is an important member of the cytochrome oxidase $\mathrm{P} 450$

\footnotetext{
* Correspondence: jintianbo@gmail.com Xue $\mathrm{He}$ and $\mathrm{Na} \mathrm{He}$ are joint first author.

'Key Laboratory for Basic life science Research of Tibet autonomous region School of Medicine, Xizang Mingzu University, Xianyang, Shaanxi 712082, China

${ }^{2}$ Key laboratory for molecular genetic mechanisms and intervention research on high altitude disease of Tibet autonomous region, School of Medicine, Xizang Mingzu University, Xianyang, Shaanxi 712082, China

Full list of author information is available at the end of the article
}

enzyme system, and polymorphisms in CYP450 enzymes are responsible for observed variation in drug responses among patients of different ethnic origins. The CYP2D6 gene located at chromosome 22q 13.2 is one of the most polymorphic CYP450 genes. It contains nine exons and eight introns, has a full-length base coding sequence of $1491 \mathrm{bp}$, expresses about 497 amino acids, and is involved in the metabolism of $20-25 \%$ of clinical prescription drugs [4]. For instance, Debrisoquine, Antidepressants,Tricyclic antidepressants, Beta-blockers et al. It has a high degree of genetic variation. To date, more than 300 variants of the CYP2D6 gene have been identified (http://www.cypalleles.ki.se), although multiple allele identified no function [5]. CYP2D6*3, CYP2D6*4, CYP2D6*5, and CYP2D6*6 are reported to be associated with decreased enzymatic activity, CYP2D6 * 4 is the most common mutants in Caucasians. About $5 \%$ to $10 \%$ and $1 \%$ are slow metabolism in Caucasians and Asians respectively. Three common mutant alleles related with reduced catalytic activity, CYP2D $6 * 10$ were found mainly in Asians, CYP2D6*17 were found mainly in African. CYP2D6* 41 were found in African and 
Caucasians. In addition to fast metabolizers, more than $5 \%$ of Caucasians are classified as ultra-fast metabolizers, Because of multiple copies in the CYP2D6 gene which enhances the ability of metabolism [6]. For example, seven percent of Caucasoid and two to seven percent of Negroid individuals are poor metabolizers of drugs dependent on CYP2D6, which metabolizes many beta blockers, antidepressants, opioids, and other compounds [7]. The Uygur population, comprising 10,069,346 individuals (The Sixth National Census), lives almost entirely within the Xinjiang Uygur Autonomous Region, Northwestern frontier area of China, represents a typical admixture population with a genetic background of Caucasians (40 \%) and East Asians (60\%) $[8,9]$. At present, there is no comprehensive system for the study of CYP2D6 polymorphisms in the Uygur population of China. So to establish a database of CYP2C9 allele frequencies for the healthy Uygurs, which would be useful for personalized medicine, we decided to systematically screen the polymorphisms of the CYP2C9 gene and compared their allelic frequencies with previous data of other ethnic groups. We hoping to identify characteristics of the genetic polymorphisms of CYP2D6 and provide reasonable recommendations pertaining to the safer administration of drugs dependent on CYP2D6 in the Uygur population.

\section{Methods}

\section{Study subjects}

We recruited a random sample of 96 healthy, unrelated Uygur individuals (48 males and 48 females) between November 2014 and January 2015 from the Tibet Nationality College in Xianyang for genetic polymorphism research. All of the chosen subjects were volunteers living in the Xinjiang Uygur Autonomous Region of China and had at least three generations of Uygur paternal ancestry. Our research adopted strict recruitment and exclusion rules. We excluded individuals with chronic diseases, conditions involving vital organs (lung, heart, kidney, brain, and liver), and several metabolic endocrinological, metabolic, and nutritional diseases. The purpose of the exclusion procedures was to minimize the known environmental and therapeutic factors that influence genetic variation in the genes of interest.

We informed all of the participants of the experimental procedures and the purpose of the study. The Human Research Committee for the Approval of Research Involving Human Subjects at the Xizang Mingzu University approved the use of human tissue in this study. We also obtained signed, informed consent from each study participant.

\section{PCR and DNA sequencing}

Genomic DNA was extracted from $300 \mu \mathrm{l}$ peripheral blood using a GoldMagMini Whole Blood Genomic DNA
Purification Kit (GoldMag Ltd). The purity of the extracted DNA reached above $99 \%$. Our PCR primers, which were described in a previous study, were designed to amplify 2000 bp of the 5' flanking regions, all exons, and all introns of the CYP2D6 gene [10]. The $10 \mu \mathrm{l} \mathrm{PCR}$ system contained $5 \mu \mathrm{l}$ Hotstar Taq Master Mix, $1 \mu \mathrm{l}$ genomic DNA (20 ng/ $\mu \mathrm{l}), 0.5 \mu \mathrm{l}$ each primer pair $(5 \mu \mathrm{M})$, and $3 \mu \mathrm{l}$ deionized water. The PCR reaction conditions were as follows: the thermal profile consisted of denaturation at $95{ }^{\circ} \mathrm{C}$ for $15 \mathrm{~min}$, followed by 35 cycles of denaturation at $95{ }^{\circ} \mathrm{C}$ for $30 \mathrm{~s}, 60{ }^{\circ} \mathrm{C}$ for $30 \mathrm{~s}$, and extension at $72{ }^{\circ} \mathrm{C}$ for $1 \mathrm{~min}$, followed by a final extension at $72{ }^{\circ} \mathrm{C}$ for $3 \mathrm{~min}$ and subsequent storage at $4{ }^{\circ} \mathrm{C}$. We detected the PCR products by agarose gel electrophoresis and directly sequenced them using an ABI Prism BigDye Terminator Cycle Sequencing Kit version 3.1 on an ABI Prism 3100 sequencer (Applied Biosystems).

\section{Data analysis}

The CYP2D6 variants were named based on the nucleotide reference sequence AY545216 (http:www.cypalleles.ki.se/) and the protein reference sequence P10635. The allelic and genotypic frequencies were calculated by a statistical method. Comparisons of allelic frequencies among different geographic populations or other ethnic populations were done using chi-squared tests with the significance level set at 0.05 . Our study used Haploview 4.1 to assess linkage disequilibrium (LD) and Hardy-Weinberg equilibrium for each genetic variant [11]. We constructed haplotypes from selected tag SNPs and derived the haplotype frequencies for the Uygur population.

\section{Functional prediction}

To analyze variants in the exon regions of CYP2D6, our study adopted the online tool PolyPhen2 (http://genetics.bwh.harvard.edu/pph2/) and SIFT (http://sift.bii.astar.edu.sg/) to predict the protein function of missense mutations, which could change the function of the cytochrome P450 enzyme. Each variant was evaluated based on the impact of protein function. The PolyPhen2 output was divided into five categories: probably benign (0.000$0.999)$, borderline (1.000-1.249), potentially damaging (1.250-1.449), possibly damaging (1.500-1.999) and probably damaging $(\geq 2.000)$. The protein function predicted by two aspects included HumVar and HumDiv.. The SIFT output was divided into four categories: tolerant (0.201$1.00)$, borderline (0.101-0.20), potentially intolerant (0.051-0.10) and intolerant (0.00-0.05).

\section{Results}

Genetic variants

We successfully identified CYP2D6 polymorphisms in 96 healthy, unrelated Uygur volunteers from the Tibet Nationality College. We identified a total of 62 CYP2D6 
Table 1 The frequencies and positions of CYP2D6 genetic variants in the Uygur population

\begin{tabular}{|c|c|c|c|c|c|c|}
\hline SNP & Allele & Position & Nucleotide change & Frequency & Region & Amino-acid effect \\
\hline rs1080989 & & -1000 & $R(G>A)$ & $46.67 \%$ & Promoter & No translated \\
\hline rs28624811 & & -740 & $Y(C>T)$ & $59.38 \%$ & Promoter & No translated \\
\hline rs 28633410 & & -678 & $R(G>A)$ & $59.38 \%$ & Promoter & No translated \\
\hline rs74966855 & & -498 & $M(C>A)$ & $5.21 \%$ & Promoter & No translated \\
\hline rs1080992 & & -365 & $R(G>A)$ & $4.17 \%$ & Promoter & No translated \\
\hline / & & -334 & $S(G>C)$ & $1.04 \%$ & Promoter & No translated \\
\hline rs34167214 & & -331 & $\mathrm{~K}(\mathrm{~T}>\mathrm{G})$ & $1.04 \%$ & Promoter & No translated \\
\hline \multirow[t]{2}{*}{ rs35534760 } & & -328 & $Y(C>T)$ & $1.04 \%$ & Promoter & No translated \\
\hline & & -327 & $R(A>G)$ & $1.04 \%$ & Promoter & No translated \\
\hline / & & -321 & $S(C>G)$ & $1.04 \%$ & Promoter & No translated \\
\hline / & & -320 & $R(A>G)$ & $1.04 \%$ & Promoter & No translated \\
\hline / & & -203 & $Y(C>T)$ & $1.04 \%$ & Promoter & No translated \\
\hline / & & -202 & $R(G>A)$ & $1.04 \%$ & Promoter & No translated \\
\hline / & & -97 & $R(G>A)$ & $1.04 \%$ & Promoter & No translated \\
\hline rs769258 & & 31 & $R(G>A)$ & $3.16 \%$ & Exon1 & Val11Met \\
\hline rs1065852 & CYP2D6*10 & 100 & $Y(C>T)$ & $45.26 \%$ & Exon1 & Pro34Ser \\
\hline rs146558635 & & 123 & $M(C>A)$ & $1.05 \%$ & Exon1 & Pro41 $=S M$ \\
\hline rs1080995 & & 214 & $S(G>C)$ & $59.38 \%$ & Intron 1 & No translated \\
\hline rs1080996 & & 221 & $M(C>A)$ & $59.38 \%$ & Intron 1 & No translated \\
\hline rs74644586 & & 223 & $S(C>G)$ & $59.38 \%$ & Intron 1 & No translated \\
\hline rs76312385 & & 227 & $Y(T>C)$ & $59.38 \%$ & Intron 1 & No translated \\
\hline rs75276289 & & 232 & $S(G>C)$ & $61.46 \%$ & Intron 1 & No translated \\
\hline rs28695233 & & 233 & $M(A>C)$ & $45.83 \%$ & Intron 1 & No translated \\
\hline rs56011157 & & 245 & $R(A>G)$ & $45.83 \%$ & Intron 1 & No translated \\
\hline / & & 254 & $K(G>T)$ & $1.04 \%$ & Intron 1 & No translated \\
\hline rs29001678 & & 270 & $Y(C>T)$ & $2.08 \%$ & Intron 1 & No translated \\
\hline rs28371699 & & 310 & $K(G>T)$ & $84.38 \%$ & Intron 1 & No translated \\
\hline / & & 317 & $R(A>G)$ & $1.05 \%$ & Intron 1 & No translated \\
\hline / & & 323 & $R(G>A)$ & $1.05 \%$ & Intron 1 & No translated \\
\hline rs28371701 & & 745 & $S(C>G)$ & $57.29 \%$ & Intron 1 & No translated \\
\hline rs71328650 & & 842 & $\mathrm{~K}(\mathrm{~T}>\mathrm{G})$ & $57.29 \%$ & Intron 1 & No translated \\
\hline rs28371703 & CYP2D6*48 & 973 & $M(C>A)$ & $14.58 \%$ & Exon 2 & Leu91Met \\
\hline rs28371704 & & 983 & $R(A>G)$ & $14.58 \%$ & Exon 2 & His94Arg \\
\hline rs28371705 & & 996 & $S(C>G)$ & $16.67 \%$ & Exon 2 & Thr98 = SM \\
\hline rs1081003 & CYP2D6*10 & 1038 & $Y(C>T)$ & $31.25 \%$ & Exon 2 & Phe112=SM \\
\hline rs368389952 & & 1070 & $R(G>A)$ & $1.04 \%$ & Intron 2 & No translated \\
\hline rs1081004 & & 1169 & $R(G>A)$ & $4.17 \%$ & Intron 2 & No translated \\
\hline rs1058164 & CYP2D6*10 CYP2D6 *39 & 1662 & $S(G>C)$ & $77.08 \%$ & Exon 3 & Val136 = SM \\
\hline novel & & 1673 & $R(G>A)$ & $1.04 \%$ & Exon 3 & Arg140His \\
\hline novel & & 2467 & $Y(T>C)$ & $7.29 \%$ & Exon 5 & Leu231Pro \\
\hline novel & & 2471 & $Y(T>C)$ & $9.38 \%$ & Exon 5 & $\mathrm{His} 232=\mathrm{SM}$ \\
\hline rs28371718 & & 2576 & $M(C>A)$ & $9.38 \%$ & Exon 5 & Pro267 $=$ SM \\
\hline novel & & 2607 & $R(G>A)$ & $100.00 \%$ & Exon 5 & Glu278Lys \\
\hline rs201830078 & & 2611 & $W(T>A)$ & $9.38 \%$ & Exon 5 & Met279Lys \\
\hline
\end{tabular}


Table 1 The frequencies and positions of CYP2D6 genetic variants in the Uygur population (Continued)

\begin{tabular}{|c|c|c|c|c|c|c|}
\hline rs76015180 & & 2662 & $R(G>A)$ & $1.09 \%$ & Intron 5 & No translated \\
\hline rs28371722 & & 2664 & $R(G>A)$ & $6.52 \%$ & Intron 5 & No translated \\
\hline rs187203531 & & 2721 & $S(G>C)$ & $1.09 \%$ & Intron 5 & No translated \\
\hline rs28371726 & & 3255 & $Y(T>C)$ & $1.04 \%$ & Exon 7 & $\mathrm{His} 361=\mathrm{SM}$ \\
\hline novel & & 3273 & $S(G>C)$ & $1.04 \%$ & Exon 7 & Giy367 = SM \\
\hline / & & 3350 & $K(G>T)$ & $1.04 \%$ & Intron 7 & No translated \\
\hline rs1985842 & & 3385 & $M(C>A)$ & $58.33 \%$ & Intron 7 & No translated \\
\hline rs28578778 & & 3394 & $Y(T>C)$ & $3.13 \%$ & Intron 7 & No translated \\
\hline rs28371729 & & 3436 & $M(C>A)$ & $2.08 \%$ & Intron 7 & No translated \\
\hline rs2004511 & & 3583 & $R(A>G)$ & $44.79 \%$ & Intron 7 & No translated \\
\hline rs28371730 & & 3585 & $R(G>A)$ & $59.38 \%$ & Intron 7 & No translated \\
\hline rs28371731 & & 3791 & $Y(C>T)$ & $59.38 \%$ & Intron 7 & No translated \\
\hline rs28371732 & & 3829 & $R(G>A)$ & $1.04 \%$ & Exon 8 & Ser401 = SM \\
\hline rs769157652 & CYP2D6*27 & 3854 & $R(G>A)$ & $13.54 \%$ & Exon 8 & Glu410Lys \\
\hline rs1135840 & CYP2D6*10 CYP2D6 *39 & 4181 & $S(G>C)$ & $100.00 \%$ & Exon 9 & Ser486Thr \\
\hline / & & 4375 & $Y(C>T)$ & $1.04 \%$ & 3'UTR & No translated \\
\hline rs116390392 & & 4482 & $R(G>A)$ & $59.38 \%$ & $3^{\prime} U T R$ & No translated \\
\hline rsr35028622 & & 4723 & $K(T>G)$ & $61.40 \%$ & 3'UTR & No translated \\
\hline
\end{tabular}

The position is according to the reference sequence AY545216 in Genbank; Not translated: this SNP has no effect on the protein sequence; UTR means untranslated region; SM means synonymous mutation

polymorphisms in the current Uygur population which contained 16 novel found SNP. Among 62 Uygur CYP2D6 polymorphisms, there were 19 amino-acid effects which contained nine synonymous mutations and ten nonsynonymous mutations, and five of which were novel. The synonymous mutations included $2471 \mathrm{~T}>\mathrm{C}$ and 3273G > $\mathrm{C}$, and the non-synonymous mutations included 1673G > A, $2467 \mathrm{~T}>\mathrm{C}$ and $2607 \mathrm{G}>\mathrm{A}$ (Table 1).

\section{Allelic frequencies and genotypic frequencies}

We identified five CYP2D6 alleles in the Uygur population. The CYP2D6*1 allele had the highest frequency (47.4\%) and represented the wild-type CYP2D6 allele, which was followed by the CYP2D6*39 allele (22.9\%) and the CYP2D6*10 allele (15.6\%). The other two alleles, CYP2D6*27 and *48, were relatively rare. The CYP2D6 allelic frequencies within the Uygur population are shown in Table 2.

We also detected five CYP2D6 genotypes, with frequencies ranging from 5.2 to $35.4 \%$ in the Uygur

Table 2 Allelic frequencies of CYP2D6 in the Uygur population

\begin{tabular}{lllc}
\hline Allele & Total $(n=192)$ & Phenotype & Frequency (\%) \\
\hline${ }^{* 1}$ & 91 & Normal & 47.4 \\
${ }^{* 10}$ & 30 & Decreased & 15.6 \\
$* 27$ & 13 & Normal & 6.8 \\
$* 39$ & 44 & Normal & 22.9 \\
$* 48$ & 14 & Normal & 7.3 \\
\hline
\end{tabular}

population. The heterozygous genotype $* 1 /{ }^{*} 10$ (31.3\%) led to a decrease in enzyme activity, while the other four genotypes, including *1/*27 (13.5\%), "1/"39 (35.4\%), "1/ "48 (14.6\%), and the rare homozygous *39/*39 (5.2\%), did not affect the enzyme activity. According to the Haploview analysis, all of the allelic and genotypic frequencies fit Hardy-Weinberg equilibrium. The CYP2D6 genotypic frequencies are shown in Table 3.

We further compared the CYP2D6 allelic frequencies between the Uygur population and other ethnic populations from various countries. The frequencies of CYP2D6*10, "27, and *39 in the Uygur population were different from those in the other ethnic groups (Table 4).

\section{Linkage disequilibrium analysis}

We adopted the Haploview software to perform LD analysis with confidence intervals to define blocks. LD is the population-genomic feature used in genetic association studies to find the location of variants that predispose individuals to genetic diseases [12]. The D'

Table 3 Genotypes of CYP2D6 in the Uygur population

\begin{tabular}{lllc}
\hline Genotype & Total $(n=96)$ & Phenotype & Frequency (\%) \\
\hline${ }^{*} 1 /{ }^{*} 10$ & 30 & Decreased & 31.3 \\
${ }^{*} 1 /{ }^{*} 27$ & 13 & Normal & 13.5 \\
${ }^{*} 1 /{ }^{*} 39$ & 34 & Normal & 35.4 \\
${ }^{*} 1 /{ }^{*} 48$ & 14 & Normal & 14.6 \\
$* 39 / *^{*} 39$ & 5 & Normal & 5.2 \\
\hline
\end{tabular}


Table 4 CYP2D6 allelic frequencies in the Uygur population

\begin{tabular}{llllllll}
\hline Population & Total number & \multicolumn{3}{l}{ CYP2D6 frequency (\%) } & Reference \\
\cline { 3 - 6 } & & $*^{*} 1$ & $* 10$ & $* 27$ & $* 39$ & \\
\hline Uygur & 96 & 47.4 & 15.6 & 6.8 & 22.9 & \\
UAE & 151 & 39.1 & $3.3^{* *}$ & $0.7^{* *}$ & $4.0^{* *}$ & {$[28]$} \\
Korean & 400 & $33.25^{* *}$ & $45.0^{* *}$ & $0.38^{* *}$ & $0.63^{* *}$ & {$[29]$} \\
Japanese & 206 & 43.0 & $38.1^{* *}$ & $0.2^{* *}$ & $0.3^{* *}$ & {$[30]$} \\
Brazilian & 873 & 39.9 & $2.05^{* *}$ & 0 & $0.8^{* *}$ & {$[31]$} \\
Chinese & 400 & $24.65^{* *}$ & $52.53^{* *}$ & 0 & 0 & {$[10]$} \\
Han & & & & & & \\
Caucasian & 330 & $35.5^{*}$ & $2.7^{* *}$ & 0 & 0 & {$[32]$} \\
Spanish & 105 & $31^{*}$ & $1.9^{* *}$ & 0 & 0 & {$[33]$} \\
Austrian & 93 & 34.9 & $4.3^{* *}$ & 0 & 0 & {$[34]$} \\
Sardinian & 250 & $31.4^{* *}$ & $5.4^{* *}$ & 0 & 0 & {$[35]$} \\
\hline
\end{tabular}

Note: ${ }^{* *} p<0.01 ;{ }^{*} p<0.05$; the $p$ value means the comparison of the allelic frequencies between the Uygur population and other populations

value indicated the extent of LD between these SNPs which shown in Fig. 1. We identified two LD blocks from our 62 Uygur polymorphisms. The first block included two very tightly correlated markers: $842 \mathrm{~T}>$ $\mathrm{G}$ and $745 \mathrm{C}>\mathrm{G}$. The second block had a very strong linkage among 310G $>\mathrm{T}, 245 \mathrm{~A}>\mathrm{G}, 233 \mathrm{~A}>\mathrm{C}, 232 \mathrm{G}>$ C, $227 \mathrm{~T}>\mathrm{C}, 223 \mathrm{C}>\mathrm{G}, 221 \mathrm{C}>\mathrm{A}, 214 \mathrm{G}>\mathrm{C}, 100 \mathrm{C}>$ $\mathrm{T},-678 \mathrm{G}>\mathrm{A}$ and $-740 \mathrm{C}>\mathrm{T}$.

\section{Non-synonymous mutation effects}

The functional consequence of the novel nonsynonymous mutations be predicted by computationally construct the structure of mutant protein in Fig. 2. We used PolyPhen-2 and SIFT to predict the function of CYP2D6 which carried the ten different non-synonymous mutations. First, we verified CYP2D6*10 $(100 \mathrm{C}>\mathrm{T})$ is the mutant with possibly damaging the enzyme activity and CYP2D6*48 $(973 \mathrm{C}>\mathrm{A})$ is another. Then we predicted the protein function of 3 novel amino-acid change and found 1673G $>\mathrm{A}, 2467 \mathrm{~T}>\mathrm{C}$ and $2607 \mathrm{G}>\mathrm{A}$ is benign to the protein function. From the result of SIFT, we found that the results is not consistent with PolyPhen-2'. The results of SIFT shown in Table 5 and Fig. 3 shown the predicted results from PolyPhen-2.

\section{Discussion}

The CYP2D6 gene has been considered very difficult for genotypic analysis, because of its numerous polymorphisms including SNPs, gene deletions, and duplications. To date, multifarious studies have analyzed CYP2D6 genetic polymorphisms. Baclig et al. found that the allelic frequencies of CYP2D6*10 among healthy Filipino volunteers were similar to those among other Asians but markedly different from those among Caucasian populations [13]. We determined that CYP2D6"10, the most

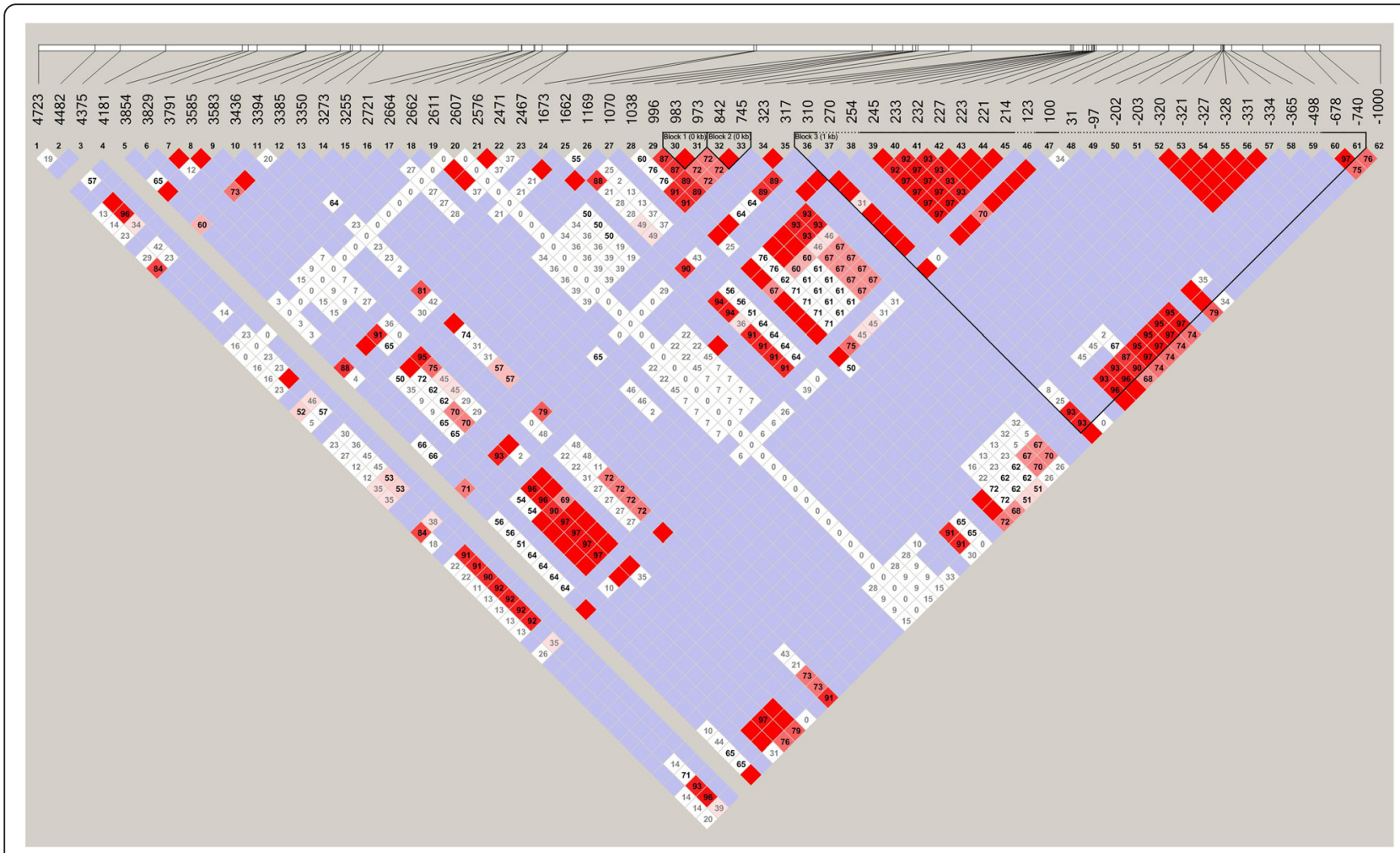

Fig. 1 Linkage disequilibrium analysis of CYP2D6 genetic polymorphisms. Strong LD is displayed by bright red (very strong: $L O D>2, D^{\prime}=1$ ) or pink red (moderately strong: $\left.L O D>2, D^{\prime}<1\right)$, intermediate $L D$ is displayed by blue $\left(L O D<2, D^{\prime}=1\right)$, and absence of $L D$ is displayed by white $\left(L O D<2, D^{\prime}<1\right)$ 

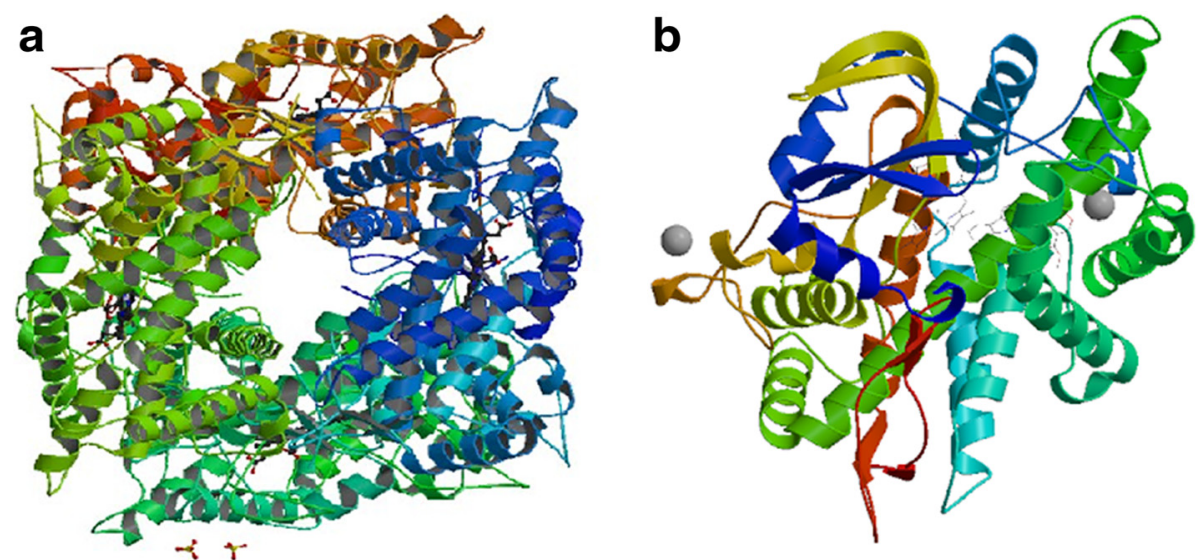

Fig. 2 Predicted computationally construct the structure of mutant protein. a Crystal Structure of Human Cytochrome P450 2D6. b CYP2D6*10

prevalent allele reported in the Asian population, had a frequency of only $15.6 \%$ among Uygur individuals. Zuo et al. found that the frequency of the CYP2D6*10 allele was not significantly different among the Han, Mongolian, or Hui populations; although the Uighur population showed significantly lower frequencies of that allele compared with the other three populations [14]. Several drugs, such as hydrocodone, are metabolized by cytochrome 450 enzymes [15], so we recommended that the dosage of that drug for Uygur individuals should be less than that used for members of the other three populations.

There were marked differences in the CYP2D6 allelic frequencies among populations with different continental origins. Some alleles were observed at high frequencies in different populations, such as CYP2D6*4 in Europeans and CYP2D6*17 in Africans $[16,17]$. For the analysis of the genetic variants of CYP2D6 in the Uygur population, there were $19 \mathrm{mu}-$ tations, comprising nine synonymous mutations and ten missense mutations. Six novel missense mutations were located at the transcription site and were predicted to result in a loss of enzyme function. Only one novel mutation was located in the 3'UTR and was therefore not translated, and we did not find any mutations in the promoter region. The CYP2D6*27 allelic frequency was $6.8 \%$ in the Uygur population; its isoforms all showed active codeine metabolism and dextromethorphan demethylation [18]. We also found a high frequency of CYP2D6*39 (22.9\%) in the Uygur population, which was different from that in the other ethnic groups and had not been previously described in the Uygur population. The CYP2D6*39 allele was previously reported to be common in Asian populations and to possibly decrease the expression level of the CYP2D6 protein; however, that allele could not transform the protein function [19].

To date, more than 50 clinically important drug substrates of CYP2D6 have been reported [20], including codeine [17], dextromethorphan [21], cyclophosphamide [22], tamoxifen [23], and ethylmorphine. There has been a lack of research to provide information regarding the influence of CYP2D6*10 on the metabolic activity of CYP2D6 in the Uygur population

Table 5 Results of SIFT predictions of non-synonymous SNPS

\begin{tabular}{|c|c|c|c|c|}
\hline$\overline{S N P}$ & Substitution & dbSNP & Score & Prediction \\
\hline $31 \mathrm{G}>\mathrm{A}$ & Val11Met & rs769258 & 0.12 & TOLERATED \\
\hline $100 C>T$ & Pro34Ser & rs1065852 & 0.00 & AFFECT PROTEIN FUNTION \\
\hline $973 \mathrm{C}>\mathrm{A}$ & Leu91Met & rs28371703 & 0.01 & AFFECT PROTEIN FUNTION \\
\hline $983 A>G$ & His94Arg & rs28371704 & 0.35 & TOLERATED \\
\hline $1673 G>A$ & Arg140His & novel & 0.00 & AFFECT PROTEIN FUNTION \\
\hline $2467 \mathrm{~T}>\mathrm{C}$ & Leu231Pro & novel & 0.11 & TOLERATED \\
\hline $2607 G>A$ & Glu278Lys & novel & 0.06 & TOLERATED \\
\hline $2611 \mathrm{~T}>\mathrm{A}$ & Met279Lys & rs201830078 & 0.00 & AFFECT PROTEIN FUNTION \\
\hline $3854 G>A$ & Glu410Lys & rs769157652 & 0.14 & TOLERATED \\
\hline $4181 G>C$ & Ser486Thr & rs1135840 & 0.37 & TOLERATED \\
\hline
\end{tabular}



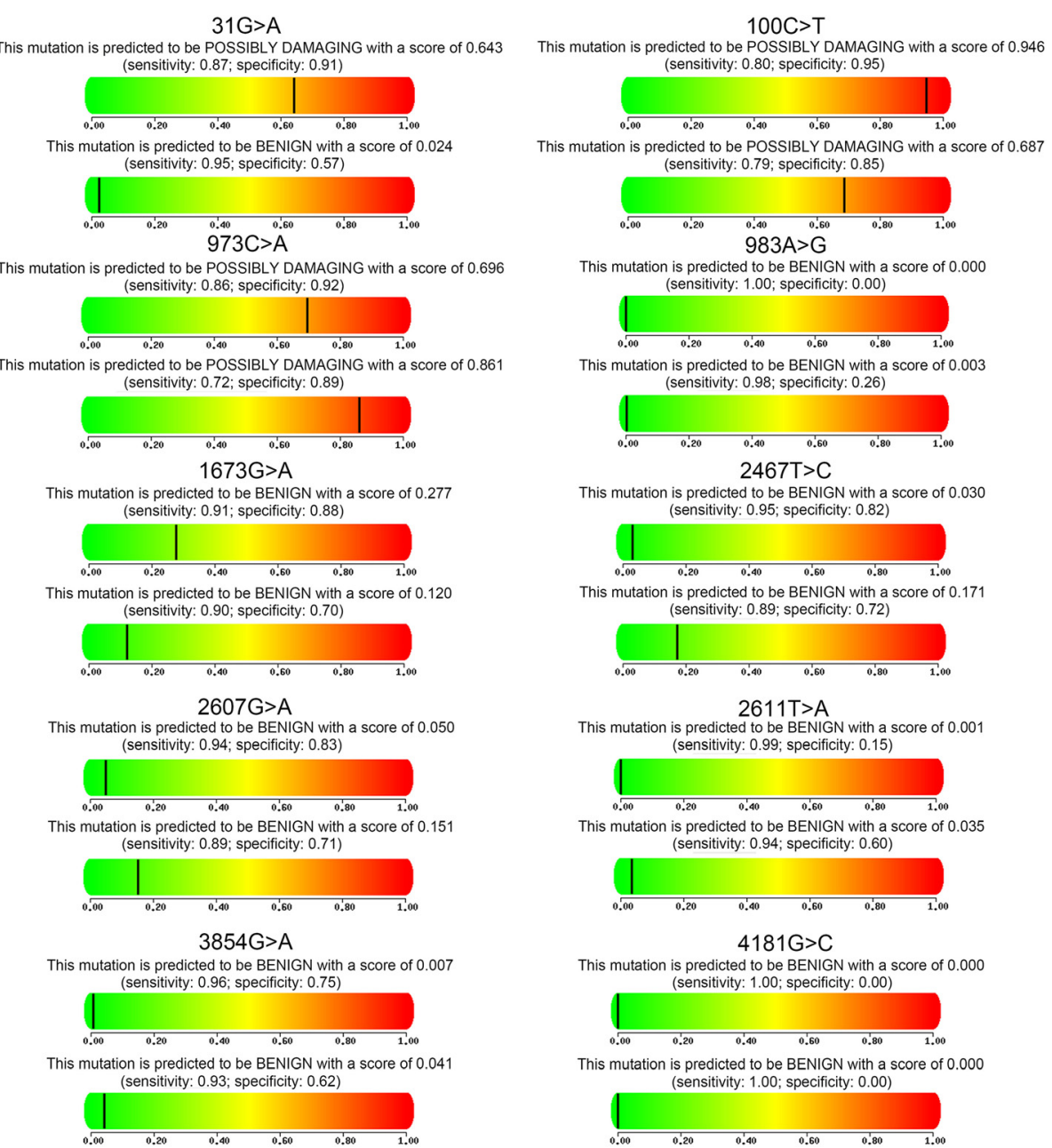

Fig. 3 Predicted protein function of all the CYP2D6 nonsynonymous mutations

[24]. Hamzeiy et al. [25] determined that CYP2D6*10 occurred more frequently in Iran $(9 \%)$ than in the UAE (3.3\%). Our study provides new data on CYP2D6 gene polymorphisms in the Uygur population and compares the frequencies of polymorphisms between the Uygur population and other ethnic populations. The differences in allelic frequencies indicate that the genetic composition also varies between the different geographical populations. That variation could contribute significantly toward a better understanding of CYP2D6 polymorphisms and to the development of a database for personalized medicine in the Uygur population. Britzi et al. [26] found a significant difference in the distribution of the metabolic ratio of the "extensive metabolizer" phenotype among Ethiopian, Russian, and Yemenite populations.

We also investigated the haplotype and LD pattern construction of the CYP2D6 gene in the Uygur population. The LD provided a basic profile of the genomic structure of CYP2D6 in the Uygur population.
Our study analyzed the pattern of LD in CYP2D6 among the Uygur and identified two blocks. We chose to deselect the loci that did not fit HardyWeinberg equilibrium to ensure that our further analyses produced reliable results. In order to see the differences in the LD structure, we constructed the haplotypes from the tag SNPs, so the haplotype structure and distributions were different in different populations. The combined genotypic effects of some decreased-function variants would result in inactive enzymes. Combinations of different polymorphisms might produce markedly different results in terms of CYP2D6 activity.

There are some limitations in our study must be noted. The sample size of this study is relatively small. The number of patients in several genotypic groups was small when the samples were divided into different groups according to the genotype, which could influence the study results. However, the current study possessing enough power. 
Overall, we determined that about $30 \%$ of Uygur individuals had genotypes associated with decreased enzyme activity, while about $70 \%$ of the people in our research had genotypes associated with normal enzyme activity. A previous study found some relationships among haplotypes and certain diseases, drug clearance rates, and adverse drug reactions, involving several joint mutation sites that reduced the functions of the enzyme [27]. Different polymorphic sites and interactions led to significant differences in enzyme activity, and haplotype analysis was more beneficial to the identification of metabolic phenotypes.

\section{Conclusion}

In summary, our research systematically analyzed the variants of CYP2D6 by directly sequencing that gene in members of the Uygur population and comparing the results with those from other ethnic populations around the world. Our work offers some useful information for the establishment of a database of CYP2D6 genetic polymorphisms in the Uygur population, which would provide a theoretical basis for individualized medical treatment and drug genomics studies. Next, we will concentrate on identifying CYP2D6 variants in a larger sample size of Uygur individuals in order to benefit the advancement of personalized medicine.

\section{Abbreviations}

CYP: Cytochrome P450; LD: Linkage disequilibrium.

\section{Acknowledgments}

This work was funded by the Major science and technology research projects of Xizang (Tibet) Autonomous Region (2015XZ01G23) and National Natural Science Foundation of China (No. 81560516)

\section{Availability of supporting data}

Other information is provided as supplementary files.

\section{Authors' contributions \\ Xue He and $\mathrm{Na}$ He carried out the molecular genetic studies. Lisong Ren participated in the sequence alignment and drafted the manuscript. Yongri Ouyang participated in the sequence alignment. Tianbo Jin, Ning Zhang and Yini Ma participated in the design of the study and performed the statistical analysis. Tianbo Jin and Longli Kang conceived of the study. Dongya Yuan helped to draft the manuscript. All authors read and approved the final manuscript.}

\section{Competing interests}

The authors declare that they have no competing interests.

\section{Deposition of data}

Not applicable.

\section{Author details}

${ }^{1}$ Key Laboratory for Basic life science Research of Tibet autonomous region School of Medicine, Xizang Mingzu University, Xianyang, Shaanxi 712082, China. ${ }^{2}$ Key laboratory for molecular genetic mechanisms and intervention research on high altitude disease of Tibet autonomous region, School of Medicine, Xizang Mingzu University, Xianyang, Shaanxi 712082, China. ${ }^{3}$ National Engineering Research Center for Miniaturized Detection Systems, Xi'an, Shaanxi 710069, China. ${ }^{4}$ School of Life Sciences, Northwest University, Xi'an, Shaanxi 710069, China.
Received: 6 September 2015 Accepted: 11 May 2016

Published online: 26 May 2016

\section{References}

1. Wilkinson GR. Drug metabolism and variability among patients in drug response. N Engl J Med. 2005;352(21):2211-21.

2. Slaughter RL, Edwards DJ. Recent advances: the cytochrome P450 enzymes. Ann Pharmacother. 1995;29(6):619-24.

3. Ji L, Pan S, Wu J, Marti-Jaun J, Hersberger M. Genetic polymorphisms of CYP2D6 in Chinese mainland. Chin Med J. 2002;115(12):1780-4.

4. Koski A, Ojanperä I, Sistonen J, Vuori E, Sajantila A. A fatal doxepin poisoning associated with a defective CYP2D6 genotype. Am J Forensic Med Pathol. 2007;28(3):259-61.

5. Sosa-Macías M, LLerena A. Cytochrome P450 genetic polymorphisms of Mexican indigenous populations. Drug Metabol Drug Interact. 2013;28(4): 193-208.

6. Ingelman-Sundberg M, Sim SC, Gomez A, Rodriguez-Antona C. Influence of cytochrome P450 polymorphisms on drug therapies: pharmacogenetic, pharmacoepigenetic and clinical aspects. Pharmacol Ther. 2007;116(3):496-526.

7. Kamal NNSBNM, Lim TS, Tye GJ, Ismail R, Choong YS. The Effect of CYP2B6, CYP2D6, and CYP3A4 Alleles on Methadone Binding: A Molecular Docking Study. Am J Chem Soc. 2013;2013:1-7.

8. Xu S, Huang W, Qian J, Jin L. Analysis of genomic admixture in Uyghur and its implication in mapping strategy. Am J Hum Genet. 2008;82(4):883-94.

9. Yao Y-G, Kong Q-P, Wang C-Y, Zhu C-L, Zhang Y-P. Different matrilineal contributions to genetic structure of ethnic groups in the silk road region in china. Mol Biol Evol. 2004;21(12):2265-80.

10. Jin T-B, Ma L-F, Zhang J-Y, Yuan D-Y, Sun Q, Zong T-Y, Geng T-T, Cui Y-L, Kang $\mathrm{L}-\mathrm{L}$, Chen C. Polymorphisms and phenotypic analysis of cytochrome P450 2D6 in the Tibetan population. Gene. 2013;527(1):360-5.

11. Barrett J, Fry B, Maller J, Daly M. Haploview: analysis and visualization of LD and haplotype maps. Bioinformatics. 2005;21(2):263-5.

12. Zondervan KT, Cardon LR. Designing candidate gene and genome-wide case-control association studies. Nat Protoc. 2007;2(10):2492-501.

13. Baclig MO, Predicala RZ, Mapua CA, Lozano-Kühne JP, Daroy M, Natividad FF, Javier FO. Allelic and genotype frequencies of catechol-Omethyltransferase (Val158Met) and CYP2D6* 10 (Pro34Ser) single nucleotide polymorphisms in the Philippines. IntJ Mol Epidemiol Genet. 2011;3(2):115-21.

14. Zuo J, Xia D, Jia L, Guo T. Genetic polymorphisms of drug-metabolizing phase I enzymes CYP3A4, CYP2C9, CYP2C19 and CYP2D6 in Han, Uighur, Hui and Mongolian Chinese populations. Die Pharmazie-An Int J Pharm Sci Res. 2012;67(7):639-44.

15. Jin Y, Desta Z, Stearns V, Ward B, Ho H, Lee K-H, Skaar T, Storniolo AM, Li L, Araba A. CYP2D6 genotype, antidepressant use, and tamoxifen metabolism during adjuvant breast cancer treatment. J Natl Cancer Inst. 2005;97(1):30-9.

16. Zhou S. Polymorphism of human cytochrome P450 2D6 and its clinica significance: part II. Clin Pharmacokinet. 2009;48(12):761-804.

17. Crews KR, Gaedigk A, Dunnenberger HM, Leeder JS, Klein TE, Caudle KE, Haidar CE, Shen DD, Callaghan JT, Sadhasivam S. Clinical Pharmacogenetics Implementation Consortium guidelines for cytochrome P450 2D6 genotype and codeine therapy: 2014 update. Clin Pharmacol Ther. 2014;95(4):376-82.

18. Zhang $W-Y$, Tu Y-B, Haining RL, Yu A-M. Expression and functional analysis of CYP2D6. 24, CYP2D6. 26, CYP2D6. 27, and CYP2D7 isozymes. Drug Metab Dispos. 2009;37(1):1-4.

19. Saruwatari J, Nakashima H, Tsuchimine S, Nishimura M, Ogusu N, Yasui-Furukori N. Possible impact of the CYP2D6* 10 polymorphism on the nonlinear pharmacokinetic parameter estimates of paroxetine in Japanese patients with major depressive disorders. Pharm Pers Med. 2014;7:121

20. Ota T, Kamada Y, Hayashida M, Iwao-Koizumi K, Murata S, Kinoshita K. Combination analysis in genetic polymorphisms of drug-metabolizing enzymes CYP1A2, CYP2C9, CYP2C19, CYP2D6 and CYP3A5 in the Japanese population. Int J Med Sci. 2014;12(1):78-82.

21. Wang D, Poi MJ, Sun X, Gaedigk A, Leeder JS, Sadee W. Common CYP2D6 polymorphisms affecting alternative splicing and transcription: long-range haplotypes with two regulatory variants modulate CYP2D6 activity. Hum Mol Genet. 2014;23(1):268-78.

22. Gor PP, Su HI, Gray RJ, Gimotty PA, Horn M, Aplenc R, Vaughan WP, Tallman MS, Rebbeck TR, DeMichele A. Research article Cyclophosphamidemetabolizing enzyme polymorphisms and survival outcomes after adjuvant chemotherapy for node-positive breast cancer: a retrospective cohort study. Breast Cancer. 2010;6:8. 
23. Nakamura Y, Ratain MJ, Cox NJ, Mcleod HL, Kroetz DL, Flockhart DA. Re: CYP2D6 genotype and tamoxifen response in postmenopausal women with endocrine-responsive breast cancer: the Breast International Group 1-98 trial. J Natl Cancer Inst. 2012;104(16):1264.

24. Lee S-J, Lee SS, Jung H-J, Kim H-S, Park S-J, Yeo C-W, Shin J-G. Discovery of novel functional variants and extensive evaluation of CYP2D6 genetic polymorphisms in Koreans. Drug Metab Dispos. 2009; 37(7):1464-70.

25. Kouhi $H$, Hamzeiy $H$, Barar J, Asadi M, Omidi Y. Frequency of five important CYP2D6 alleles within an Iranian population (Eastern Azerbaijan). Genet Test Mol Biomarkers. 2009;13(5):665-70.

26. Britzi M, Bialer M, Arcavi L, Shachbari A, Kapitulnik J, Soback S. Genetic polymorphism of CYP2D6 and CYP2C19 metabolism determined by phenotyping Israeli ethnic groups. Ther Drug Monit. 2000;22(5):510-6.

27. Mikus $\mathrm{G}$. Introduction to adverse drug reactions and drug-drug interaction Therapeutische Umschau Revue therapeutique. 2011;68(1):3-9.

28. Qumsieh RY, Ali BR, Abdulrazzaq YM, Osman O, Akawi NA, Bastaki SM. Identification of new alleles and the determination of alleles and genotypes frequencies at the CYP2D6 gene in Emiratis. PLoS One. 2011;6(12):e28943.

29. Lee S-Y, Sohn KM, Ryu JY, Yoon YR, Shin JG, Kim J-W. Sequence-based CYP2D6 genotyping in the Korean population. Ther Drug Monit. 2006;28(3):382-7.

30. Sakuyama K, Sasaki T, Ujiie S, Obata K, Mizugaki M, Ishikawa M, Hiratsuka M. Functional Characterization of 17 CYP2D6 Allelic Variants (CYP2D6. 2, 10, 14A-B, 18, 27, 36, 39, 47-51, 53-55, and 57). Drug Metab Dispos. 2008;36(12):2460-7.

31. Friedrich DC, Genro JP, Sortica VA, Suarez-Kurtz G, de Moraes ME, Pena SD, dos Santos ÂKR, Romano-Silva MA, Hutz MH. Distribution of CYP2D6 alleles and phenotypes in the Brazilian population. PLoS One. 2014;9(10):e110691.

32. Rebsamen M, Desmeules J, Daali Y, Chiappe A, Diemand A, Rey C, Chabert J, Dayer P, Hochstrasser D, Rossier M. The AmpliChip CYP450 test: cytochrome P450 2D6 genotype assessment and phenotype prediction. Pharmacogenomics J. 2009;9(1):34-41.

33. Menoyo A, del Rio E, Baiget M. Characterization of variant alleles of cytochrome CYP2D6 in a Spanish population. Cell Biochem Funct. 2006; 24(5):381-5.

34. Beer B, Erb R, Pitterl F, Niederstätter H, Maroñas O, Gesteira A, Carracedo A, Piatkov I, Oberacher H. CYP2D6 genotyping by liquid chromatographyelectrospray ionization mass spectrometry. Anal Bioanal Chem. 2011:400(8):2361-70.

35. Falzoi M, Pira L, Lazzari P, Pani L. Analysis of CYP2D6 allele frequencies and identification of novel SNPs and sequence variations in Sardinians. ISRN Genet. 2012;2013:1-10

\section{Submit your next manuscript to BioMed Central and we will help you at every step:}

- We accept pre-submission inquiries

- Our selector tool helps you to find the most relevant journal

- We provide round the clock customer support

- Convenient online submission

- Thorough peer review

- Inclusion in PubMed and all major indexing services

- Maximum visibility for your research

Submit your manuscript at www.biomedcentral.com/submit 